\title{
Prevention of perinatal group B streptococcal disease: screening during a pregnancy
}

\section{Rosella Bruno, Donatella Caldera, Graziella Cacciola, Luciana Chilin, Sabrina Stumpo, Giuseppe Neri}

S.S. Microbiologia, Laboratorio Analisi Chimico Cliniche e Microbiologia, Ospedale Civico di Chivasso, ASL TO 4 Regione Piemonte

Key words: Streptococcus agalactiae, Early-onset disease, Screening pregnant women

Prevenzione delle infezioni neonatali da S. agalactiae: screening in gravidanza

\section{SUMMARY}

The prevention of perinatal group B streptococcal (GBS) disease is based on the screening of all pregnant women at 35-37 weeks' gestation for vaginal and rectal GBS colonization.

The colonized women receive intrapartum antibiotic prophylaxis.

Our study reports the different rates of maternal GBS colonization between April 2008 and March 201 I.

We have collected 3430 samples by swabbing both the lower vagina and rectum and we have employed two different laboratory methods: direct agar plating and selective enrichment broth.

The rates of maternal GBS colonization increased from 10.5\% during 2008-2009, to 12.2\% during 2009-2010 and to $14.4 \%$ during $2010-201 \mathrm{I}$, when we have introduced the Todd Hewitt broth.

Our results show that the use of an enrichment broth improves detection of GBS carriers women.

\section{INTRODUZIONE}

La sepsi neonatale da Streptococcus agalactiae (SGB) è ancora tra le principali cause di morbilità e mortalità neonatale.

L'incidenza di sepsi precoce si è ridotta con l'applicazione di protocolli per lo screening in gravidanza e la chemioprofilassi intrapartum.

Le Linee Guida del CDC di Atlanta (2010) prevedono l'esecuzione di tampone vaginale e rettale alla $35 \mathrm{a}-37 \mathrm{a}$ settimana di gravidanza al fine di evidenziare le gravide colonizzate da SGB, cui effettuare profilassi antibiotica al parto.

L'infezione neonatale si definisce precoce (Early Onset disease) se si manifesta entro la prima settimana di vita: nel $90 \%$ dei casi nelle prime 24 ore.

Le più comuni sindromi sono sepsi e polmonite $\mathrm{e}$ meno frequentemente meningite. Nei neonati pretermine la mortalità raggiunge il $30 \%$ dei casi di infezione.

È stimato che 1'1-2\% di neonati, nati da madri colonizzate con SGB sviluppino early onset disease.

Alcuni fattori aumentano il rischio di sepsi precoce: parto prematuro $<37$ settimane, rottura pretermine delle membrane, febbre intrapartum $>38^{\circ} \mathrm{C}$ e precedente nato con infezione da GBS.

Lo scopo del nostro lavoro è valutare la frequenza di isolamento di $S$. agalactiae nei campioni esaminati presso la S.S. Microbiologia dell'Ospedale
Civico di Chivasso, anche in relazione al metodo di indagine utilizzato.

\section{METODI}

Sono stati analizzati 3430 campioni (ognuno costituito da un tampone vaginale e un tampone rettale - Transystem Liquid Stuart) dal 1 aprile 2008 al 31 marzo 2011.

I Metodo: sino al 30 marzo 2010 i campioni venivano direttamente seminati su piastre (Vacutest Kima) di Agar Columbia 5\% Sangue di Montone (tampone vaginale) e Agar Columbia 5\% Sangue di Montone addizionato con CNA (tampone rettale), poi incubate a $37^{\circ} \mathrm{C}$ per $18-24$ ore.

II Metodo: dal 1 aprile 2010, secondo le linee di indirizzo della Regione Piemonte pubblicate nel febbraio 2010, i tamponi vaginale e rettale vengono immessi in una stessa provetta contenente brodo Todd Hewitt-CNA (Figura I).

Dopo incubazione overnight si esegue sottocoltura su piastre di Agar Columbia 5\% Sangue Montone addizionato con CNA, incubate a $37^{\circ} \mathrm{C}$ per 18-24 ore.

La conferma di positività viene effettuata mediante test sierologico (Streptococcal Grouping Latex kit - PRO-LAB) sulle colonie che presentano betaemolisi (Figura II) non emolitiche.

Data la riconosciuta sensibilità di Streptococcus agalactiae a penicillina, ampicillina e alle cefalosporine di I generazione, non viene effettuato di

\section{Corresponding author: Rosella Bruno}

S.S. MICROBIOLOGIA, Ospedale Civico di Chivasso

Corso G. Ferraris 3; 10034 Chivasso (TO) - Tel.: 0 I I 9176286

E-mail: microbiologia.chivasso@aslto4.piemonte.it 
routine il test di sensibilità agli antibiotici sui ceppi isolati, se non nei casi segnalati di allergia ai betalattamici: si procede allora a valutare la eventuale resistenza a eritromicina e a clindamicina.

\section{RISULTATI}

Dal 1 aprile 2008 al 31 marzo 2009 si sono analizzati 1024 campioni: $108(10.5 \%)$ sono risultati positivi per SGB. Dal 1 aprile 2009 al 31 marzo 2010 si sono esaminati 1173 campioni, di cui 143 $(12 \%)$ positivi.

Dal 1 aprile 2010 al 31 marzo 2011 i campioni, analizzati utilizzando il brodo di arricchimento, sono stati 1233; di questi 178 (14.4\%) sono risultati positivi (Figure III).

\section{CONCLUSIONI}

I valori di positività alle indagini di screening per la ricerca di $S$. agalactiae nelle gestanti in diverse realtà sanitarie italiane, secondo un rapporto dell'ISS, variano da $3.9 \%$ al $19.4 \%$. La percentuale di positività da noi osservata è in aumento dal 2008 ad oggi: la sensibilità dello screening risulta maggiore con l'utilizzo di arricchimento in brodo. Numerosi trials clinici evidenziano che l'antibiotico profilassi intrapartum alle donne colonizzate da SGB ha una efficacia del $86-89 \%$ nel prevenire l'early-onset disease del neonato: la penicillina rimane l'antibiotico di scelta.

Pertanto l'esecuzione di un corretto screening in gravidanza è di fondamentale importanza.

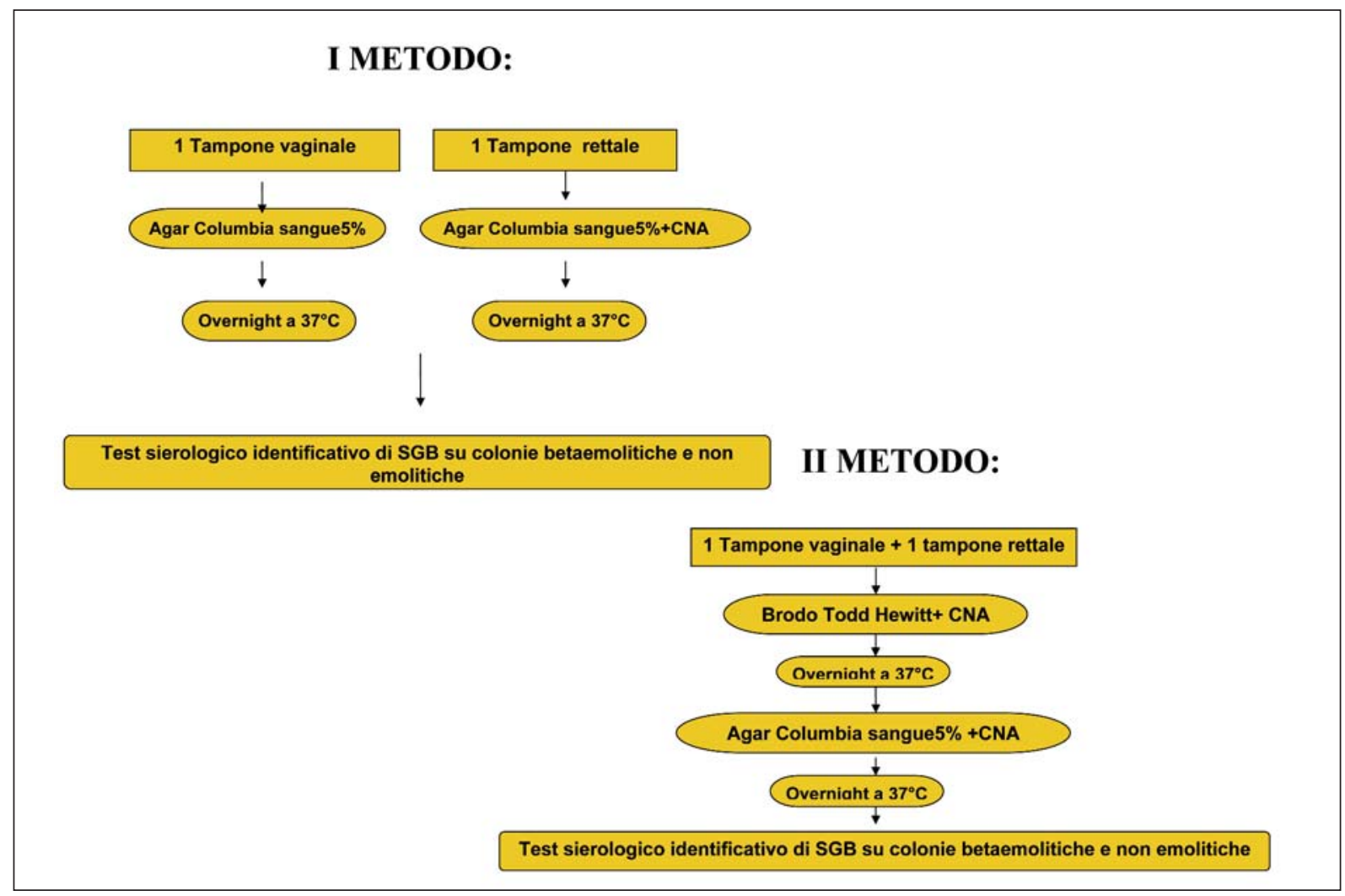

Figura I.

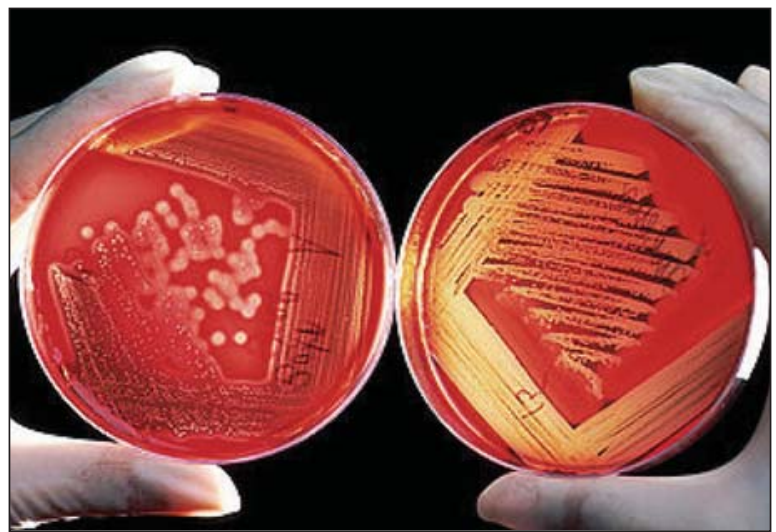

Figura II.

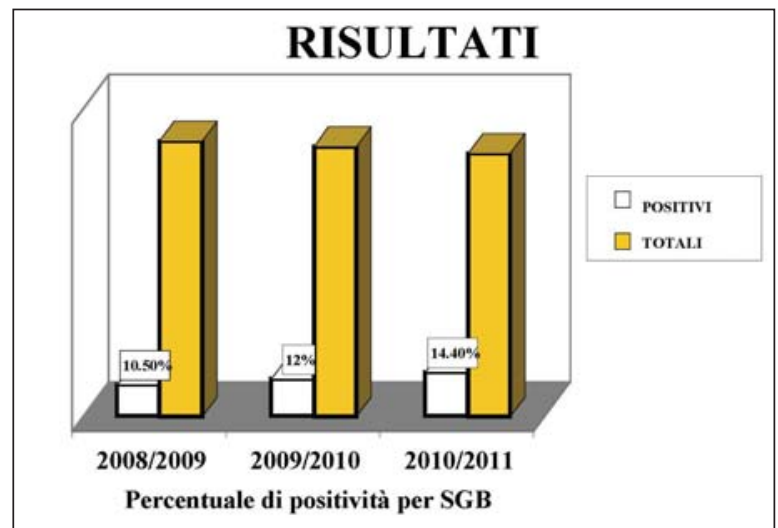

Figura III. 


\section{BIBLIOGRAFIA}

1. CDC. Prevention of Perinatal Group B Streptococcal Disease. Revised Guidelines 2010.

2. Lodolo L, Barbaglia M, et al. Indagine multicentrica sulla incidenza epidemiologica dello $S$. agalactiae in gravidanza. XXXIX Congresso AMCLI - Rimini 2010.

3. Regione Piemonte. Linee di indirizzo per la prevenzione delle infezioni neonatali precoci da Streptococco B. 18 febbraio 2011. 